\title{
Infrastructures et réseaux dans le développement économique de la Hongrie
}

In: Flux n¹0, 1992. pp. 41-51.

\begin{abstract}
Tamás FLEISCHER, Infrastructures and networks in Hungary's economic development. Technical networks and service infrastructures represent a major obstacle in the development of Hungary, according to the author. Studies have revealed lack of equipment and functional incapacity. This situation is partly the result of a long-term crisis. But it is also the reflection of the structure of centralized authority, set up after WWII, which could neither imagine nor maintain independent networks. The recent political changes, as well as expansion towards a market economy, has raised new, contradictory questions: the role of the State, methods for establishing real costs, the respective place of self-regulation and public intervention. The author feels that the solution is not to restructure the country according to the models imported from the West; rather, the public authorities still have a role to play in the control and regulation of these sectors.
\end{abstract}

\section{Résumé}

Tamás FLEISCHER, Infrastructures et réseaux dans le développement économique de la Hongrie. Les réseaux techniques et les infrastructures de services représentent un blocage majeur du développement de la Hongrie, selon l'auteur. Des enquêtes font apparaître des retards d'équipement et des dysfonctionnements. Cette situation tient pour une partie à l'héritage de longue période. C'est aussi le reflet de la structure de pouvoir centralisée mise en place après la seconde guerre mondiale qui ne peut concevoir ou maintenir des réseaux indépendants. Les changements politiques comme l'ouverture économique en direction du marché posent de nouvelles questions contradictoires: rôle de l'Etat, méthodes de mesure des coûts, places respectives de l'autorégulation et de l'intervention publique. Plutôt que de considérer que tout doit être reconstruit selon un modèle importé de l'ouest, l'auteur pense que la puissance publique a encore sa place dans la régulation de ces secteurs.

Citer ce document / Cite this document :

Fleischer Tamás, Watton Eric. Infrastructures et réseaux dans le développement économique de la Hongrie. In: Flux $n^{\circ} 10$, 1992. pp. 41-51.

doi : $10.3406 /$ flux.1992.918

http://www.persee.fr/web/revues/home/prescript/article/flux_1154-2721_1992_num_8_10_918 


\title{
INFRASTRUCTURES ET RÉSEAUX
}

\section{DANS LE DÉVELOPPEMENT ÉCONOMIQUE}

\author{
DE LA HONGRIE
}

\author{
TAMÁS FLEISCHER
}

Tamás FLEISCHER est ingénieur de l'Université Polytechnique de Budapest. Il travaille actuellement comme chercheur $\grave{a}$ The Institute for World Economics de l'Académie Hongroise des Sciences et a publié de nombreux articles et études sur l'économie des infrastructures en Hongrie.

\begin{abstract}
I'HISTOIRE de la construction des infrastructures et des $\mathbf{L}$ réseaux en Iongrie au cours du vingtième siècle fut une succession de mauvais calculs. $\Lambda$ u stade actuel de la transition économique, un danger existe du fait de l'impression prévalente que l'infrastructure peut être copiée et importée de l'Ouest comme d'autres biens de consommation. Dans cet article, nous comprenons l'infrastructure dans le sens des réseaux physiques comme les transports, les télécommunications ou l'énergie mais aussi aussi des autres réseaux comme l'éducation, la santé et le commerce, où l'organisation du système est également importante, même si les liens de ces réseaux ne sont pas physiquement visibles.
\end{abstract}

Si nous observons les différentes conceptions du développement des infrastructures nationales, nous pouvons nous apercevoir que plusicurs propositions actuelles de réforme sont fondées sur l'expérience de "bon " fonctionnement des systèmes étrangers. Bien qu'il soit évidemment intéressant d'étudier et de comprendre de tels systèmes qui fonctionnent bien, il est tout aussi important d'avoir une analyse détaillée des circonstances sur lesquelles le système national est véritablement bâti. Il est à la fois inexact et trompeur d'affirmer que l'économic hongroise est une économie de marché fonctionnant mal, que les structures héritées du développement socialiste peuvent être - une fois admis le diagnostic de faillite - condamnées et abolies et que cela suffira à provoquer d'un scul coup un nouveau départ.

\section{L'ORIGINE DES RÉSEAUX HONGROIS}

Même si nous considérons le développement des quarante années d'après-guerre, il serait injuste d'ignorer le fait que l'infrastructure de la Hongrie moderne était de nature centralisée avant cette période. La mise en place de cette 
construction remonte à une centaine d'années quand la création de la Hongrie moderne et la question de sa dépendance ou de son indépendance par rapport à l'Autriche étaient totalement liées. Budapest devait se développer comme contrepoids à Vienne. Ce processus coïncidait avec le développement du réseau ferré hongrois et cela devint un enjeu politique que de le construire aussi centré sur Budapest que possible. In fait, cette structure permit à la capitale de devenir le centre direct d'une région beaucoup plus grande qu'auparavant.

Mais, au moment même où son indépendance lui fut donnée, après la première guerre mondiale, la Hongrie perdit les deux tiers de ses territoires. Avec l'amputation de ces provinces et des villes qui formaient un deuxième niveau de développement après la capitale (sous leur dénomination présente Bratislava, Kosice, Cluj-Napoca, 'Iimisoara, Oradea, Subotica, Novi-Sad, etc.), disparurent également les lignes ferroviaires qui les reliaient entre elles. Ce changement accentua l'importance relative de la capitale.

Entre les deux guerres, alors que la ligne politique officielle était de s'accrocher à la pérennité de la Grande IIongric, aucun effort ne fut fait pour prendre en compte les nouvelles frontières. Les corrections nécessaires au réseau des transports intéricurs ne furent pas apportées. Ce qui peut être considéré comme le point de départ d'une cascade d'idées fausses.

Après 1945, la direction communiste hérita d'une structure démographique surcentralisée, mais rien ne se produisit pour la corriger. Au contraire, le nouveau pouvoir politique s'y installa tout à son aise et utilisa à son avantage les hiérarchies post-féodales existantes. Par les mesures administratives prises après 1950, Budapest s'élargit. A cette époque, $20 \%$ de la population du pays $y$ vivait, alors que la population de la plus grande ville après la capitale n'atteignait pas le dixième de celle-ci. Bien que la différence entre les 5 plus grandes villes et Budapest se soit atténuée dans les 40 années suivantes, les proportions n'ont pas changé.

Pour le réseau de transport, en septembre 1990, il y avait 42 lignes traversant le Danube qui sépare la Hongrie en deux parties, orientale et occidentale: 37 d'entre elles (voies ferrées, métro, tram compris) se trouvaient dans Budapest. Malgré cela, les projets de développements officiels donnent encore la priorité aux ponts de la capitale et aux lignes de métro avec 16 lignes supplémentaires en construction ou envisagées. Comment se fait-il que même les décisions nouvelles maintiennent la tendance d'un réseau hypercentralisé ?

Aujourd'hui, les professionnels aussi bien que les citoyens sont confrontés en permanence à la misère du téléphone, à des problèmes dans le transport public, la fourniture d'énergie et le stockage des déchets. Cette observation faite à partir d'une enquête de 1984 s'inscrit dans une lignée plus géné. rale d'analyses critiques. Au cours des quinze ou vingt dernières années, plusicurs articles furent publiés dans les revues spécialisées hongroises pour évaluer la qualité des infrastructures et les contradictions dans leur développement. Ians ces travaux, une place particulière revient au livre de Csernok, Ehrlich et Szilagyi, publić en 1975. Il résume plusicurs années de leurs travaux visant à identifier des types de développement des infrastructures à partir de l'observation d'un grand nombre de statistiques internationales. Ils montrent que le développement des infrastructures dans les économies de marché précédait ou bien allait en parallèle avec le développement économique, tandis que dans les pays socialistes il ne faisait qu'essayer de rattraper le retard sur les autres secteurs.

l.'enquête de 1984, réalisée dans les 19 départements hongrois ${ }^{1}$, permet de mettre en évidence les principaux problèmes sectoriels. Dans les transports, les problèmes les plus fréquents sont le vieillissement des gares, des centres de service pour le chemin de fer et des dépots de transbordement, tout comme leur insuffisance en termes de capacités. Sont également incriminées la mauvaise connection des routes avec les zones d'habitat et la saturation des réseaux de voiric dans et autour des villes. Revient moins souvent la nécessité d'augmenter les potenticls de trafic de certaines routes.

Une image uniforme s'est imposée pour les télécommunications. Pour 18 des 19 départements, décrire ces réscaux, c'est parler de niveau bas, de retard, d'obsolescence, de surcharge, d'état des plus critiques. Les causes de cette rareté absolue sont l'insuffisance des commutateurs et le retard dans la tech. nologie. 


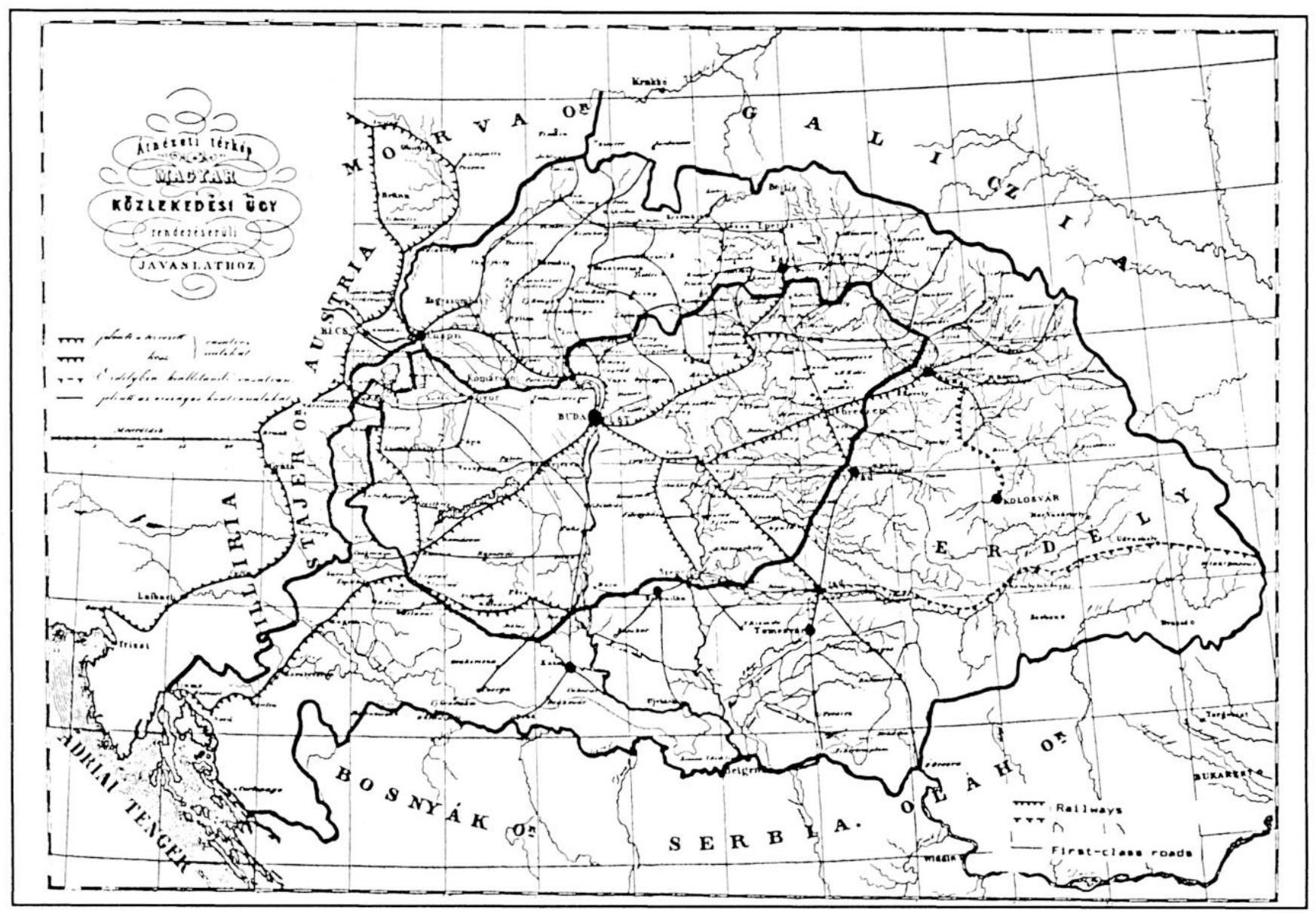

FIGURE 1 : le Programme proposé par Széchenyi pour le réscau de transports en 1848

Pour le secteur de l'eau, le plus grand problème réside dans le traitement des caux usées, avant la distribution d'eau. Pour l'énergic, il y a problème quasiment dans tous les départements pour les réseaux à moyenne et basse tension. Beaucoup ont également ajouté les insuffisances dans le réseau de distribution de gaz naturel. Globalement, il ressort de cette enquête que des problèmes sévères concernent uniformément tous les départements du pays.

\section{LA REDISTRIBUTION COORDONNÉE PAR LA MACROÉCONOMIE}

Que s'est-il passé? I.es structures de réscaux qui n'étaient pas autrefois contrôlées par le centre comme l'éducation, la santé, le commerce, furent bouleversées pendant les années cinquante pour leur donner une forme pyramidale contrôlée par un point central. I.es petites unités de commerce furent nationalisées et incluses dans un système de distribution monopolistique de grandes surfa. ces ; les écoles religieuses et privées furent fermées et une autorité nationale fut crécé pour la formation de la jeunesse autour d'un programme d'éducation unique. I.es réseaux techniques gardèrent leur structure ou les développèrent. Il était plus important de développer les stations de télévision et de radio, qui assuraient une communication à sens unique, plutôt que de construire un réscau public de téléphone. Un grand nombre de réseaux téléphoniques très élaborés et prioritaires relièrent cependant les institutions d'importance stratégique (ministère de l'Intéricur, de la Défense, Électricité Hongroise, Industrie du Gaz et du Pétrole, Chemins de Fer d'ítat IIongrois, Parti Socialiste des Travailleurs Hongrois, etc.) sous la forme de réscaux hiérarchiques et centralisés 
Une économie planifiée par le centre, fondée sur la centralisation massive et l'élimination du marché, non sculement préfère mais exige un parallélisme des réseaux techniques et politiques qui se renforcent mutuellement. Si des connections horizontales existaient aux niveaux les plus bas, communications, commerce, des germes d'autonomic apparaîtraient et affaibliraient la dépendance vis à vis du pouvoir central. Pour être capable de survivre, ce pouvoir a donc eu intérêt à détruire les liens locaux. Cela fut bien sûr obtenu sous le couvert de la nécessité "d'économies d'échelle " et de la recherche de la " taille optimale", etc. Selon cette philosophic, les commerces locaux, les écoles, les dispensaires et même certains centres urbains devaient être éliminés parce que leur maintien était « irrationnel ». I)es routes locales et des lignes téléphoniques étaient nécessaires mais il n'y avait pas "suffisamment " de ressources financières pour les construire. Il y avait toujours des objectifs "plus importants " à long terme et seuls les distributeurs des ressources centralisées en comprenaient la nécessité.
Une caractéristique naturelle de ce système est l'abolition de l'économic de marché et en contrepartic l'établissement d'un système artificiellement construit de taxes et de prix. L'ítat déclare exclusivement sienne la tâche de ravitailler les citoyens; en conséquence, les salaires ne prennent pas en compte les coûts de production du logement, du transport et de l'alimentation de base. Pour cette raison, la distribution centralisée de tous les services se fait d'un côté et le flot incontrôlé et illimité des demandes se poursuit de l'autre, ce qui, en dernière analyse, est considéré par le pouvoir comme une justification de la nécessité de centraliser la distribution.

Il est bien connu depuis la fin des années soixante que l'approche hongroise du développement économique affirmant comme position idéologique la priorité de la production comme seule source de valeur ajoutéc pour forcer l'investissement dans l'industric (et surtout dans l'industrie lourde), éloigna les ressources de la nation du développement des réseaux

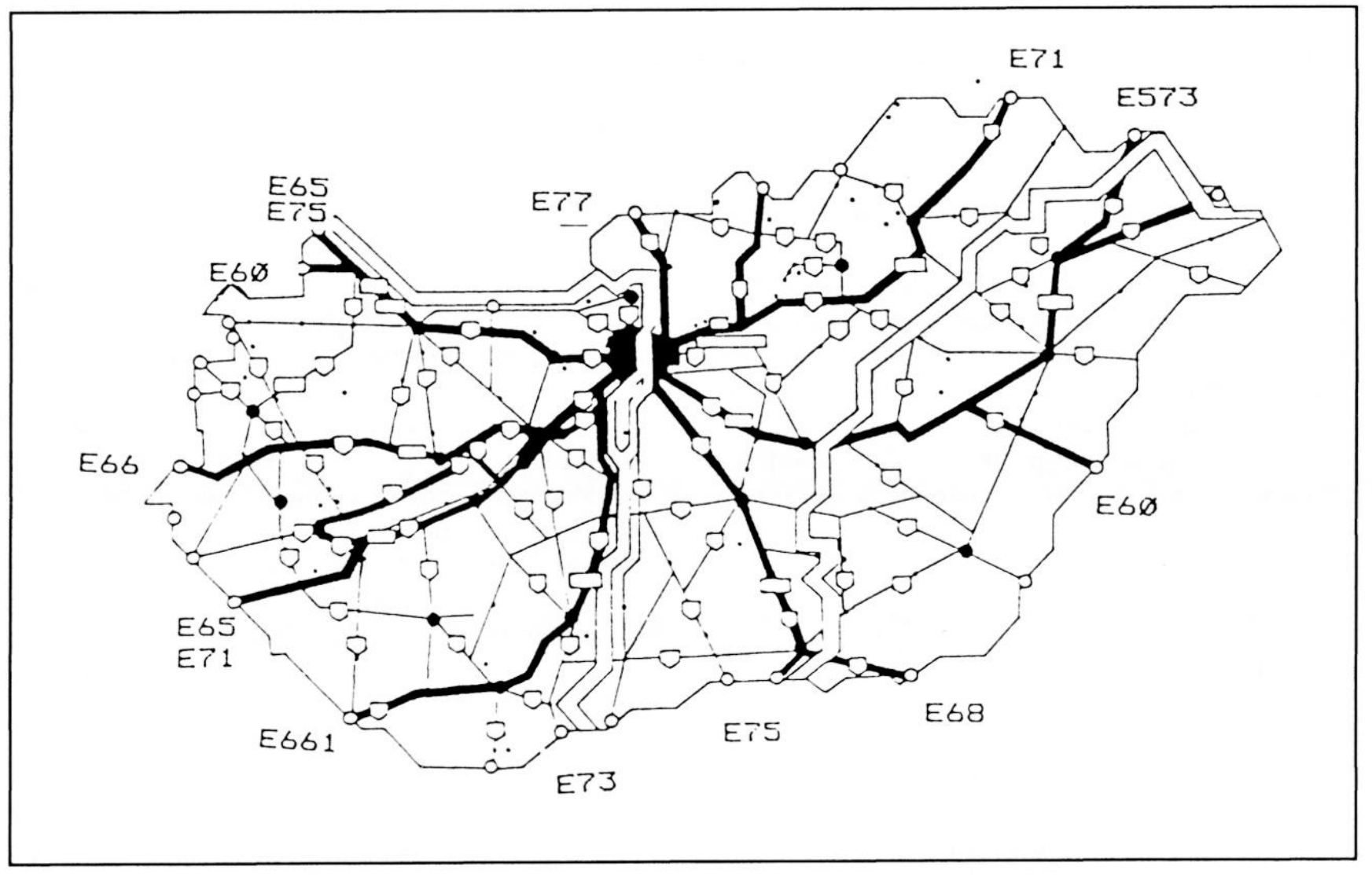

FIGURE 2 : le réseau routier.

Toutes les routes de première classe traversent le Danube à Budapest. 


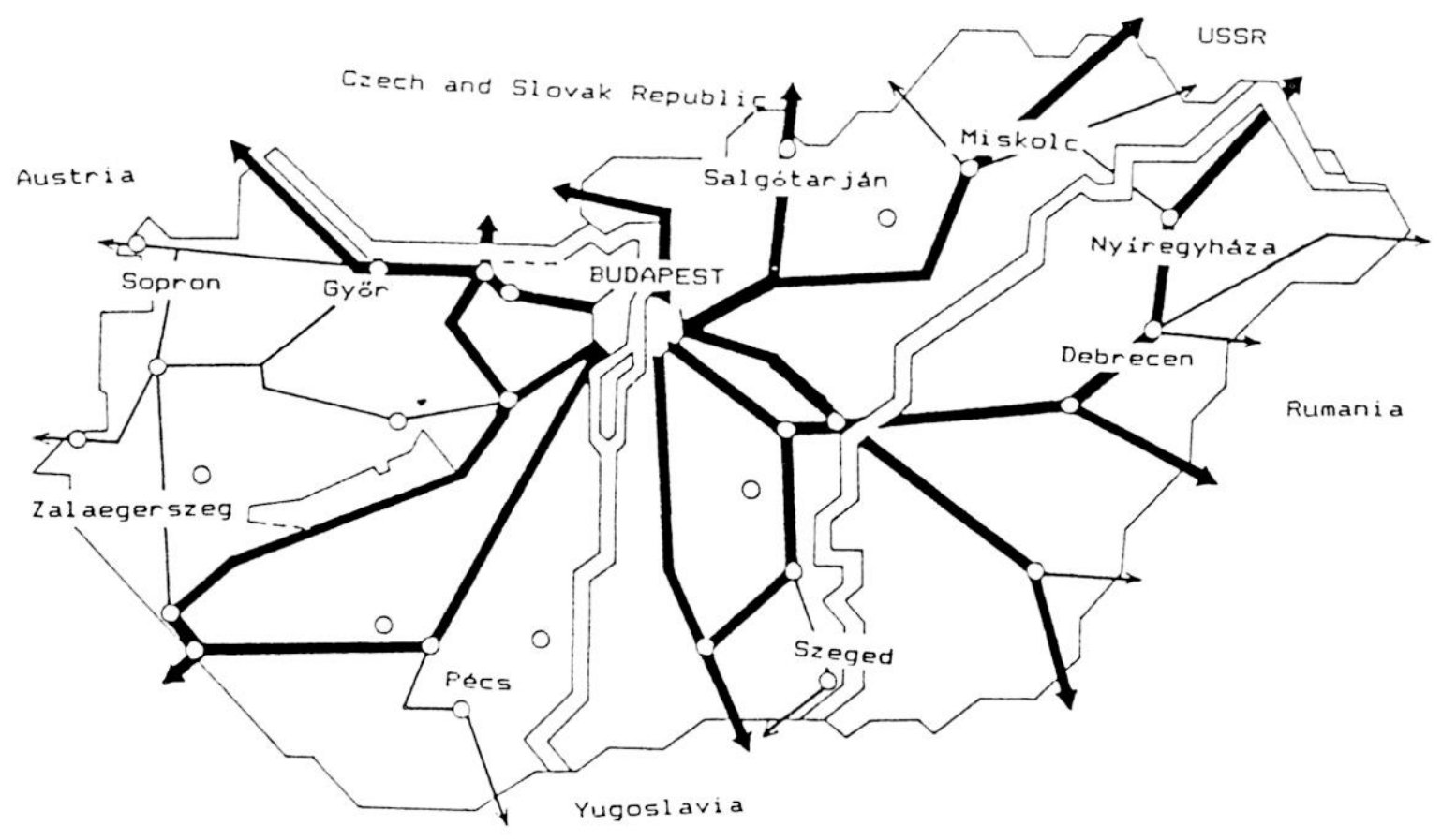

FIGURE 3: Le réseau de base du chemin de fer hongrois.

la structure est la même que pour les routes.

d'infrastructures existants et des besoins de la consommation. I.es critiques de cet état de fait servirent depuis les années soixante-dix de point de référence dans les négociations du plan intersectoriel alors que les branches de l'infrastructure (transports, communications, éducation, santé) exigeaient une plus grande part des ressources budgétaires.

\section{L'INSUFFISANCE DES \\ RESSOURCES : " PLUS D'ARGENT POUR LES INFRASTRUCTURES "}

Au même moment, une description plus objective des conditions réelles de l'économie apparut en Hongrie dans les rapports économiques publiés pour chaque secteur. Alors que dans les rapports précédents seuls les résultats étaient présentés (quel type d'établissement avait été construit et mis en fonction), les rapports les plus typiques de cette nouvelle décade donnèrent un plus grand rôle aux " descriptions les plus franches de la situation réelle ". Ces rapports faisaient la liste des problèmes dévoilés par les établissements publics et leurs filiales : les moyens de production étaient obsolètes, le remplacement avait été négligé, des pannes se produisaient régulièrement et la panne générale menaçait en permanence. Dans tous ces rapports, il était suggéré ou carrément écrit que la filière économique n'avait pas eu assez d'argent.

Si quelqu'un lisait le rapport d'une de ces filières, il pouvait croire très facilement que le budget avait été négligé dans ce cas particulier. S'il prenait le temps de lire tous les rapports de toutes les branches de léconomic, il lui paraissait impossible de croire que l'éducation comme la santé, les transports comme le réseau de distribution d'eau, les produits manufacturés et l'agriculture avaient tous été négligés de la même manière, et qu'en même temps, la métallurgie et les mines, le bâtiment et la production d'énergie avaient aussi besoin d'investissements, parce qu'ils 
avaient été si longtemps oubliés avant l'avènement du système communiste.

Il est très clair d'après cet exemple qu'il ne s'agissait pas d'un problème d'infrastructures. Pour cette raison, le slogan d'après lequel la solution pour obtenir de meilleures infrastructures était d'obtenir plus d'argent, n'avait aucun fondement, même si la nécessité peut en être prouvée.

\section{OBTENIR DES FONDS : LE CHANTAGE À LA PANNE}

Une analyse plus approfondic de ces rapports rend possible la compréhension du rôle des infrastructu. res dans l'économie nationale. Un usage hiérarchisé, hypercentralisé et surpolitisé du pouvoir et les mé. canismes macroéconomiques qui centralisaient et redistribuaient les ressources économiques aboutissaient logiquement à l'impuissance de l'économie toute entière. le résultat des mécanismes montrés plus haut aboutit à ce qu'au lieu de se concentrer sur le marché très réduit 't sur le's consommateurs, les firmes comme les divers secteurs se concentrèrent presque exclusivement sur la part de l'enveloppe budgétaire à laquelle ils prétendaient. Donc, les stratégies de survice étaient aussi liées au maintien ou à l'accroissement de la part de ressources financières de la firme dans le processus de distribution. Ceux qui prouvaient qu'ils étaient indispensables à l'économie nationale pouvaient espérer attirer l'attention du distributeur central. Plus sévèré était la menace de banqueroute, plus grandes étaient les chances de s'assurer des ressources suffisantes. Une manière d'obtenir des fonds était de pratiquer le chantage à la panne. Ians cette compétition, curicusement, la branche qui risquait de causer le tort le plus visible par son effondrement obtenait la plus grande priorité, et ainsi, faisait de ses victimes potentielles, ses alliés. İn accord avec cette liste de priorités, le secteur de l'énergie et l'industrie lourde étaient mieux placés que les industries de manufacture et/ou l'agriculture. Parmi les services, les réseaux de service produc. tifs avaient des protecteurs plus influents que les réscaux de consommateurs.

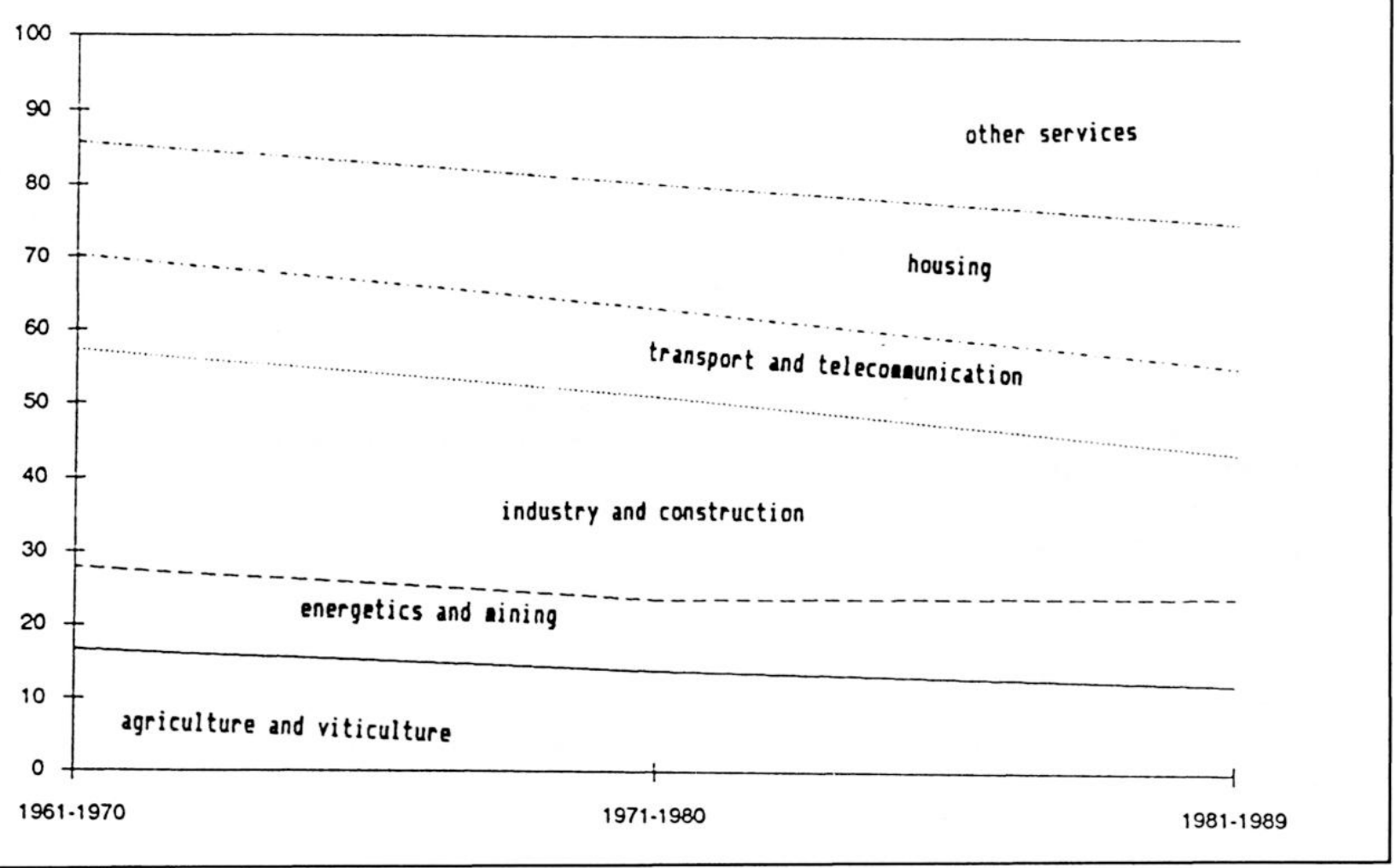

FIGLRE 4 : Investissements par secteur, $1961-1989$ (voir Table 1, p. 48) 


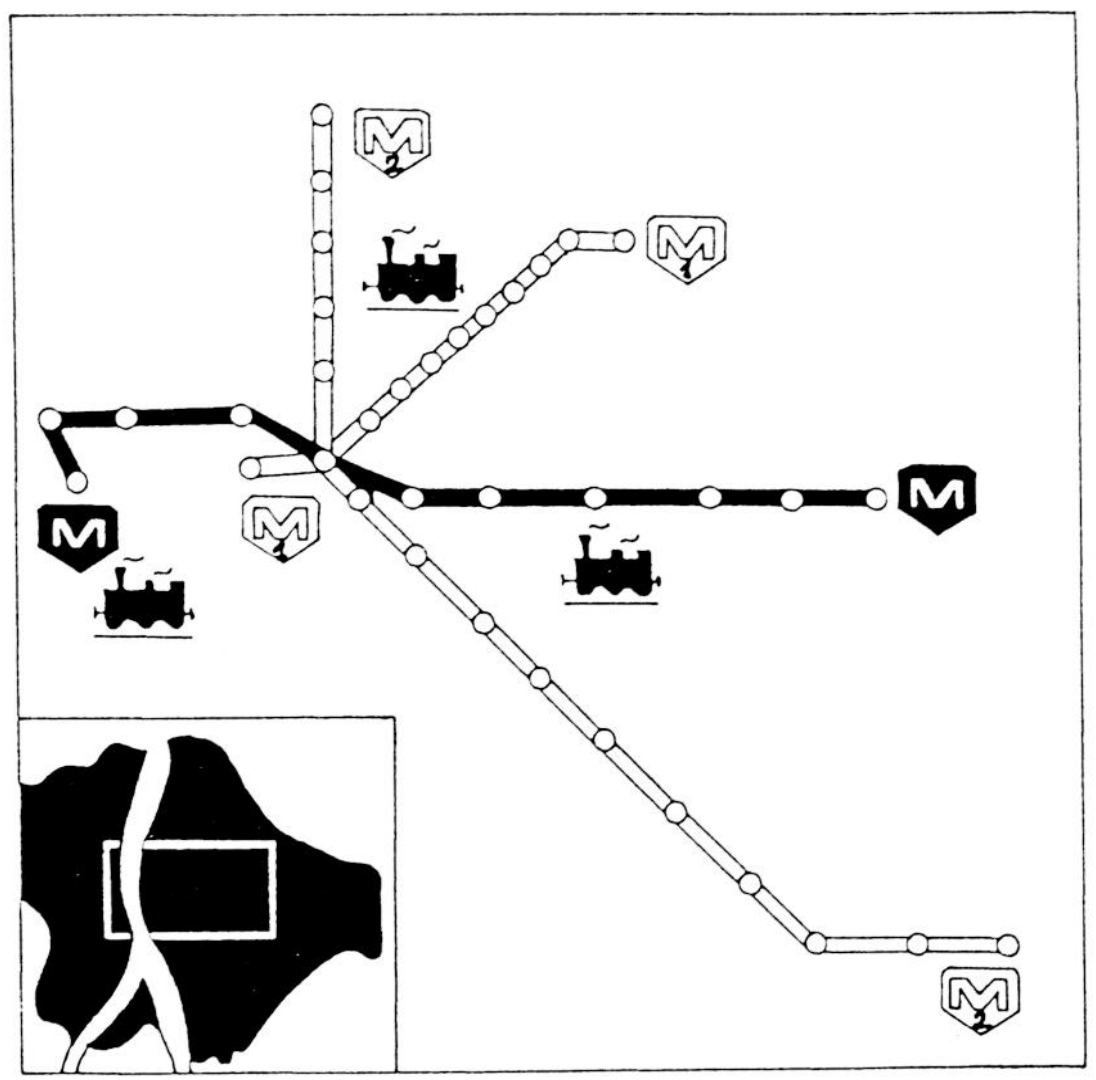

FIGURE 5 : Le souterrain à Budapest.

La réduction du problème des infrastructures dans la logique de la ligne économique redistributive révèle non seulement le problème de la redistribution de l'argent entre les différents secteurs économiques mais aussi celui de distribuer les fonds à l'intérieur même d'une branche ou d'une autre. Les structures du pouvoir centralisé et le comportement de ceux, tout en bas de l'échelle, ont aussi un impact sur la distribution ultérieure des ressources. Non seulement des pans entiers de l'industrie étaient défavorisés mais à l'intéricur de chaque branche, les consommateurs ou le secteur des services étaient tenus en disgrâce.

Fn même temps, il est important de noter qu'un pouvoir hićrarchique était établi dans chaque bran. che économique: éléments radiaux de différents réscaux (centre - centre régional - sous-centre), radio centrale à sens unique, réseaux de télévision, lignes prioritaires de téléphone et centres de commerce en gros. La hiérarchie était un élément impor tant des réseaux d'éducation et de santé et ne concernait pas seulement le secteur de la défense et les industries stratégiques connexes. Ia logique des réseaux hiérarchisés dominc aussi le modèle centralisé du développement régional, et nécessairement, reflète également dans la géographic la structure du pouvoir.

Pour revenir à la question de la distribution, on ne peut pas dire que les infrastructures en général n'eurent pas assez d'argent, mais que certains secteurs en curent et d'autres pas. Tout ce qui vient d'être dit peut être analysé de la même manière dans chaque branche et doit être mis en relation avec les mécanismes de redistribution des ressources.

\section{LA COORDINATION PAR LE MARCHÉ}

Les critiques du modèle d'économic socialiste mon. trèrent, pour plusieurs problèmes que la planification ne pouvait résoudre malgré des efforts con- 
stants, que la solution passait par “ l'autorégulation " du marché. Donc, la mission du gouverne. ment n'était pas de se substituer au marché mais d'en garantir le fonctionnement. De la même ma. nic̀re que la distribution utilisait le slogan " plus d'argent pour les infrastructures ", le principe du " plus de marché pour les infrastructures" devint dominant.

Ces idées sont dues pour une part à une réelle reconnaissance : il n'y a aujourd'hui pas de mécanismes économiques autorégulateurs qui rendraient des interventions des acteurs publics inutiles. I'un autre côté, les propositions reposent sur plusicurs concepts (comme "l'infrastruc. ture est le moteur de l'économie ") qui sont en soi vraies pour les économies de marché. Cepen. dant, personne ne peut prouver qu'ils représen. tent la force d'entraînement nécessaire pour rendre fonctionnelle l'économie. Au lieu de quoi, tout en se référant au marché et aux réformes, se produit un transfert du fardeau sur la population. C'est un mouvement unilatéral parce qu'il essaye d'engager l'argent du consommateur dans des investissements initiés par l'État sans déléguer l'autorité de décision adéquate. Un exemple typique fut la taxe de développement des agglomérations (tebo) en 1987 ; plusieurs contributions concernant les routes, les adductions et le téléphone sont gérées de la même façon. Unilatéral car, même s'il n'est pas séparé, au moins à un niveau macro-économique, du principe de lier la source de financement à l'objectif à accomplir, il n'implique pas une réforme complète des salaires qui signifierait un vrai transfert des droits de libre usage.

Les industries et les secteurs d'activités qui sont en compétition aujourd'hui pour obtenir de l'ar- gent d'unc distribution toujours centralisée se concentrent sur le client (c'est-à-dire, s'interrogent sur ses besoins et se mettent en compétition pour obtenir son argent) si les ressources prépondérantes viennent du consommatcur. Alors que si l'État finance d'importants services, par les ressources budgétaires, en maintenant les salaires au plus bas, le marché ne peut pas faire valoir ses effets réels. La correction effectuće par la redistribution du budget reste alors la seule option, au lieu de réduire le rôle de l'État à la seule correction des effets pervers du marché.

\section{INFRASTRUCTURES VENDABLES ET EXTERNALITÉS}

L'approche économique des problèmes infrastructuraux, régionaux ou environnementaux conduit au concept de la gestion et du financement au jour le jour, concept formulé généralement en termes microéconomiques. Comme cela a été présenté plus haut, les problèmes de la gestion régionale ou de la construction d'écoles étaient basés sur le rapport des calculs d'investissements; pour cette raison, la localisation et l'évaluation des ressources devinrent un sujet de calcul de profit.

Pour comprendre les effets macroéconomiques du développement infrastructurel, nous devons cesser de considérer toute l'infrastructure comme un marché. Il ne s'agit pas de nier que la majorité des activités de service, communément appelées infrastructures doivent essenticllement fonctionner dans un environnement de marché ; mais il existe une activité de création de réseau qui n'a pas de lien direct avec les calculs de profit du marché. Ce dernier est l'infrastructure dans le sens plus étroit du terme. Il

TABLE 1 : Investissements par secteur

\begin{tabular}{|l|c|c|c|}
\hline \multicolumn{1}{|c|}{ SECTEUR } & $\mathbf{1 9 6 1 - 1 9 7 0}$ & $\mathbf{1 9 7 1 - 1 9 8 0}$ & $\mathbf{1 9 8 1 - 1 9 8 9}$ \\
\hline Agriculture \& Viticulture & 16.60 & 13.80 & 12.20 \\
\hline Energie \& Mines & 11.35 & 9.83 & 11.95 \\
\hline Industrie \& Construction & 29.35 & 27.37 & 19.45 \\
\hline Autre & 42.70 & 49.00 & 56.40 \\
\hline dont: & 12.90 & 12.00 & 11.60 \\
\hline - Transports \& Communication & 15.50 & 17.20 & 19.80 \\
\hline - Construction d'habitation & & \\
\hline
\end{tabular}


est généralement accepté qu'on ne peut pas laisser sans évaluation de tels éléments.

Le point de vue économique est tellement envahissant que de tels phénomènes sont appelés extraéconomiques. Certains de ces éléments sont appelés "infrastructures " ou " industries de fond " - pour signifier qu'ils dépassent la logique du profit à la fois dans le temps et dans l'espace. Certains sont appelés externalités, pour exprimer le fait qu'ils ne tombent pas dans le système des profits financiers (le profit apparaît plus tard ou pour quelqu'un en aval, ou peut-être n'est-il pas mesurable en argent).

Les premières méthodes essayèrent d'incorporer les externalités, en traduisant chaque valeur dans une dimension unique afin de les faire rentrer dans la logique de la gestion et des profits financiers. Des analyses coût-profit tentèrent d'abord de caractériser les facteurs externes en valcurs monétaires mais ces efforts échouèrent quand il fallut exprimer les pertes dues à un accident, la valeur de la vic, celle du confort, de l'air pur, etc.

L'autre approche ne signific pas l'incorporation des facteurs externes dans un système uni-dimensionnel de profit monétaire, mais l'extension du système économique pour créer tout autour des boucles d'évitement régulatrices. C'est essenticllement la logique de l'économie de marché, régulée par l'ítat, qui réagit avec sensibilité aux flux monétaires sur une base de valeur différente, à cause de limites plus grandes.
Ce dont nous avons besoin, ce sont des cloisonnement stables, des feedbacks souples. Il est très important que les conditions de cloisonnement d'un système dirigiste suivent la logique d'un système plus lent, et donc plus stable, mais que les signaux soient ceux d'un système plus rapide.

Pour utiliser une expression mal comprise empruntée aux études sur le développement infrastructurel, nous avons un développement de type suiviste quand un système plus variable, plus capable de s'adapter détermine une structure et déforme les éléments émergeant d'un système plus lent. De cette manière, l'équilibre interne du système lent est troublé, et le système est incapable de donner assez vite les signaux. Les distorsions s'accumulent jusqu'à ce que le système s'effondre totalement. C'est ce qui se produit quand l'ítat intervient dans la production et que les réseaux régionaux et infrastructurels sont adaptés pour compléter cette production (il faut noter que ce développement ne prend pas en compte l'environnement naturel et les dotations)

\section{CONCLUSION}

Dans les pays d'Europe centrale ou de l'Est, le désintérêt pour les infrastructures avait d'abord un fondement idéologique, mais cette attitude n'a pas été modifiéc jusqu'à aujourd'hui. A la recherche de ressources, chaque branche économique utilise les armes et les arguments d'autrefois en faisant un

TABLE: 2 : la structure de l'emploi par secteur $(\%)$

\begin{tabular}{|c|c|c|c|}
\cline { 2 - 4 } \multicolumn{1}{c|}{} & \multicolumn{2}{c|}{ SECTEUR } \\
\hline ANNÉE & INDUSTRIE & AGRICULTURE & TERTIAIRE \\
\hline 1941 & 26.62 & 51.50 & 23.77 \\
\hline 1951 & 26.60 & 49.63 & 36.62 \\
\hline 1961 & 29.15 & 35.23 & 43.13 \\
\hline 1971 & 32.41 & 24.46 & 43.34 \\
\hline 1976 & 35.88 & 20.78 & 40.82 \\
\hline 1981 & 38.59 & 20.59 & 38.51 \\
\hline 1986 & 41.33 & 20.16 & 38.15 \\
\hline 1987 & 42.47 & 19.30 & 38.04 \\
\hline 1988 & 43.15 & 18.81 & 37.42 \\
\hline
\end{tabular}


chantage à la banqueroute. Si rien n'est changé et si le gaspillage continue dans les différentes branches économiques, la "modernisation" - même en acceptant l'ćconomie de marché - s'orientera dans une mauvaise direction.

le principe de la nécessaire intervention de l'État doit être fondé : 1) sur la mesure des symptômes, et 2) sur la construction des structures. Il faut se conditionner pour percevoir et évaluer les signaux mais on ne doit pas interférer à ce niveau. Un traitement aussi superficiel est inutile quand les causes sousjacentes demeurent. De cette manière, on peut justifier la nécessité de plus en plus d'interventions (ce qui peut aller dans l'intérêt des institutions ou des personnes impliquées!)

Les interférences doivent prendre en compte les changements dans les systèmes qui sont plus rapides que ceux au niveau duquel se fait l'interférence mais qui s'accordent au système ancien plus rigoureux.

Pour revenir à notre champ d'intérêt plus étroit, évidemment, on peut intervenir dans la production pour l'orienter vers le marché, mais il n'est pas pratique de le faire dans le but de provoquer des changements dans la production ou dans les structures la soutenant. La politique ayant pour but de modifier la production doit modifier son organisation systèmes régionaux et d'infrastructures - et doit laisser les détails aux mouvements internes de la sphère de production.

Les caractéristiques de l'intervention gouvernementale dans la politique régionale et infrastructurelle doivent être les suivantes :
- prise en considération de l'environne. ment naturel et de sa réaction probable (c'est l'orientation environnementale).

- connaissance des lois du développement des systèmes régionaux et infrastructuraux, qui peuvent aider à des changements positifs dans les réseaux (c'est le développement infrastructurel ou régional de type dirigiste, en d'autres termes, orientation infrastructurelle).

- reconnaissance des interrelations entre les systèmes régionaux et infrastructurels d'un côté et le système de production et des services de l'autre, reconnaissance aussi de la manière dont la produc. tion (services) répondra à un changement structurel.

- création de conditions de marché pour la production comme pour les services (orientation vers le marché)

- étude des réactions immédiates de la production.

- dans le cas de divergences ou d'effets non prévus, nécessité de revoir tout le système de relations et probablement de modifier l'action originelle au niveau de l'environnement humain.

\footnotetext{
1. La Hongrie est divisée en 19 unités administratives appelleés megye, assez semblables aux départements français. Budapest forme une vingtième unité indépendante.
} 
FIGLRF: 6 : Proportion des emplois, par secteur (voir Table 2. p. 49)

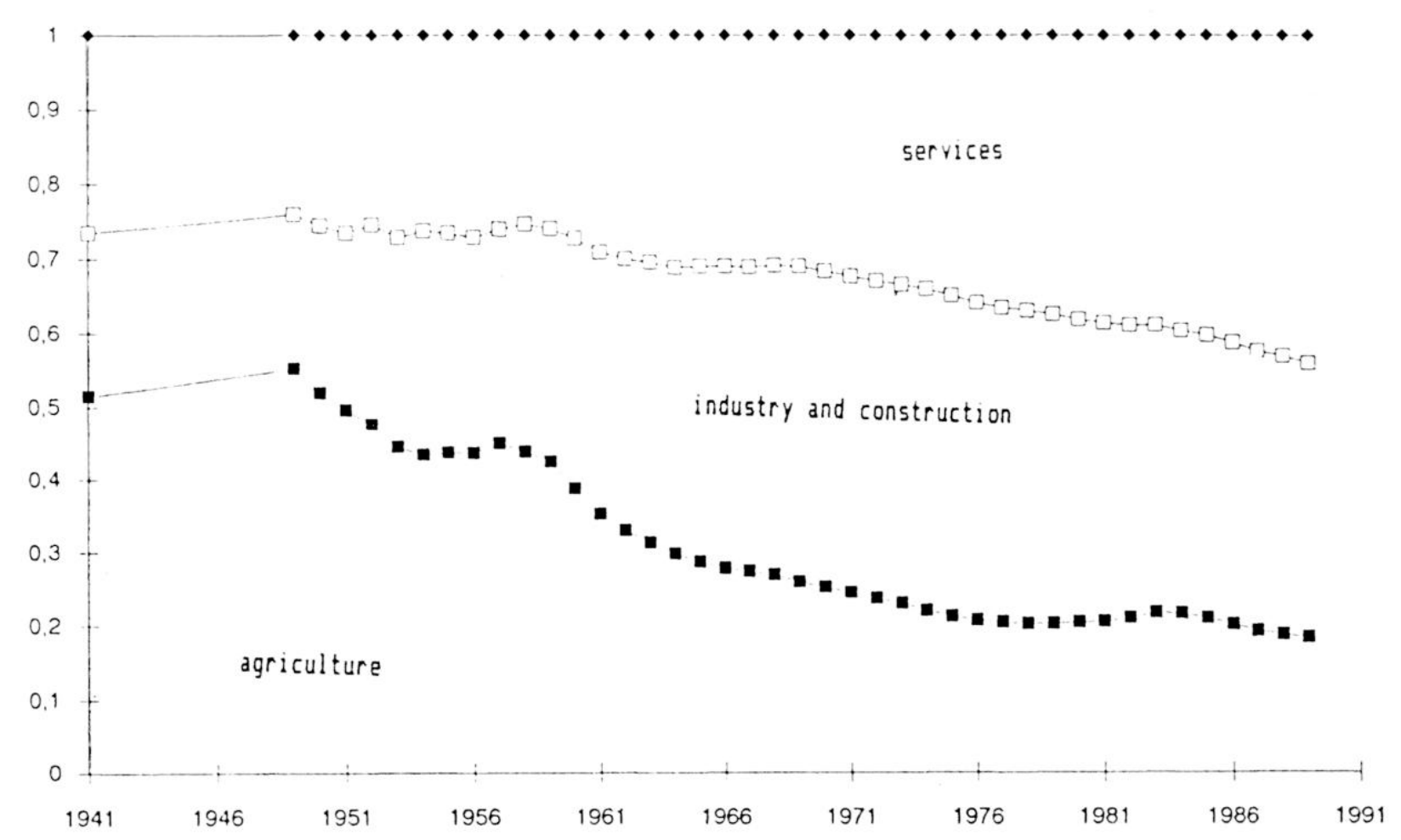

RÉFÉRENCES

CSERNOK, ATTIIA, ERIICH, FVA, \& S7IIÁGYI, GYÖRGY 1975, Infrastructura, komok, országok ("Infrastructure, périodes historiques et pays"), Kossuth Kiadó.

FlFischer, TAMÁs, 1986, "Infrastruktura fejlesztési csapkák" ("Pièges dans le développement d'infrastructures"), Közgaudasági Szemle.も

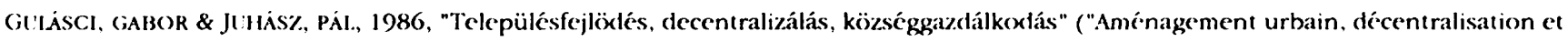
gestion des villages"), Tenugazdasági Fórum, $\mathrm{n}^{\circ} 2$.

IACKÓ, MÁRIA, 1984, "A hátrány megörzése" ("Contrôler l'évolution des stocks"), Közgazdasági Szemle, nº 3.

MAJOR, IVÁN, 1982, "Szállitási feszültségek és a közlekedés fejlettségi szintjei" ("les tensions dans les transports et le niveau de développement correspondant"), Statisztikai Szemle. $n^{\circ}$ 8-9.

MAJOR, NÀN, 1984, Közlekedés és gazdaság ("Transport et Économie"), Magvetö Kiadó.

MANDEL, MIKIÓS, LUJZA GÁSPÄR-PAP, MME \& SÄGH, GÄBOR, 1987, Infrastruktura-politika ("Politique infrastructurelle"). K̈̈zgaZdaságiés Jogi Kiadó.

SıMáx. Mıкı.os, 1983. "A szolgáltatások iráényitásának továbbfejlestéröl" ("la restructuration de la gestion des services"). Küzzaudasági Szemle, $n=2$. 